\title{
Algorithmic Issues During the FEM Mesh Preparation of Human Models Based on 3D body Scans
}

\author{
Yordan KYOSEV, Doudou ZHANG \\ TU Dresden, Institute of Textile Machinery and High Performance Material Technology (ITM), \\ Chair of Assembly Technology for Textile Products, Dresden, Germany
}

https://doi.org/10.15221/20.48

\begin{abstract}
Generating human finite element models for mechanical or thermal simulations is a complex task. Modern 3D scanning technology offers a fast method of capturing the surface of the human body and thus forms the starting point for the future creation of individual FEM models based on the 3D scan. This paper presents some frequently occurring issues and problems in mesh processing, which have often to be solved and which has to be implemented in an automatic procedure for human modelling.
\end{abstract}

Keywords: 3d body scanning, proceedings, format

\section{Introduction}

The human comfort depends on complex set of interactions between the human body, its clothing and environment. During the last years the simulation methods becomes more powerful and able to model these interactions. For such modelling, the complete geometries and material parameters of the human body, clothing and the environment have to be available. Creating FEM model of the human body is a very complex task and currently such models are only available for some fixed bodies and for certain applications like crash tests. This paper presents some algorithmic issues during the selection of the strategy and development of an algorithm for automatic FEM mesh generation based on 3D Scan data of humans.

\section{State of the art}

The automotive industry has been using FEM models of humans, called virtual dummies, for quite some time. An overview of several anthropometric models available for the LSCT software LS-Dyna can be found in [1]. These models were initially based on rigid body parts and later real elastic properties were implemented. They are suitable for simulation of the load over the safety belt and for evaluation of the accelerations over the body, but each model is based on one certain fixed geometry. The most precise method for creation of human models would be based on micro-CT scans of human body, as the used one in [2]. Actually this method requires using of complex and expensive equipment and specialized algorithms for the image processing and it is not efficient for parametric studies with different body sizes. One promising method for modelling humans using FEM method is explained in [3], where the difference of the deformation of the textiles using elastic human chest in the contrary to the commonly used in the fitting software rigid chest is demonstrated. The leading research group in the modelling of the human body is the Max Planck Institute for Intelligent Systems in Tübingen, which creates data driven algorithms for human generation, based on the large size of scanned bodies and their motion [4], [5]. None of these works provide fast algorithm for creation of FEM model of the real human, based on 3D scan with acceptable accuracy. The current work presents some of the initial steps, done in this direction, connected with the resoling problems with the processing of the scan data during the preparation for automatic generation of the FEM mesh.

\section{Mesh issues}

\subsection{From raw data to mesh}

The 3D scanners obtain during the scan process a set of points (point cloud) which describe the surface of the scanning body (Fig. $1 \mathrm{a}$ ). The distance between these points and their arrangement in rows and columns depends on the number of the cameras of the scanning system and the type of the relative motion between the object and each camera (stationary, rotation, free motion for 4D scanners). Since the final 3D data set combines the points from several 3D images, received from separate cameras and from same cameras from different relative positions to the body, the final set of the cloud points is normally not regular. In some areas there are several points, in other areas even missing points. Before 
processing this data to triangulate mesh, it is useful to remove all points $p_{j}$, which are closer to a base point $p_{i}$ than the minimal sampling distance $r_{\text {sampling }}$. Condition (1) is implemented as "filter" in most software packages and libraries

$$
\left\|p_{i}-p_{j}\right\|<r_{\text {sampling }}
$$

After removing the duplicate or the nearest points, a triangle mesh is generated, so that the entire body surface can be described as a waterproof set of triangles (Fig. 1b).

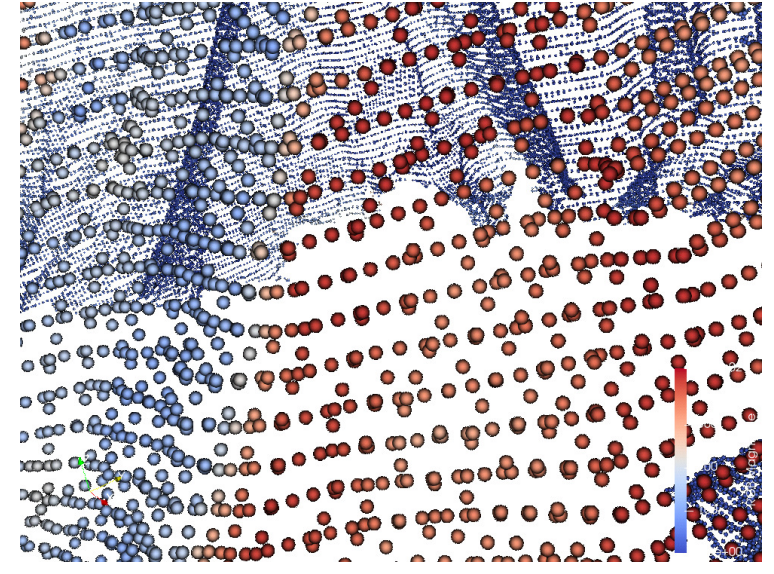

a

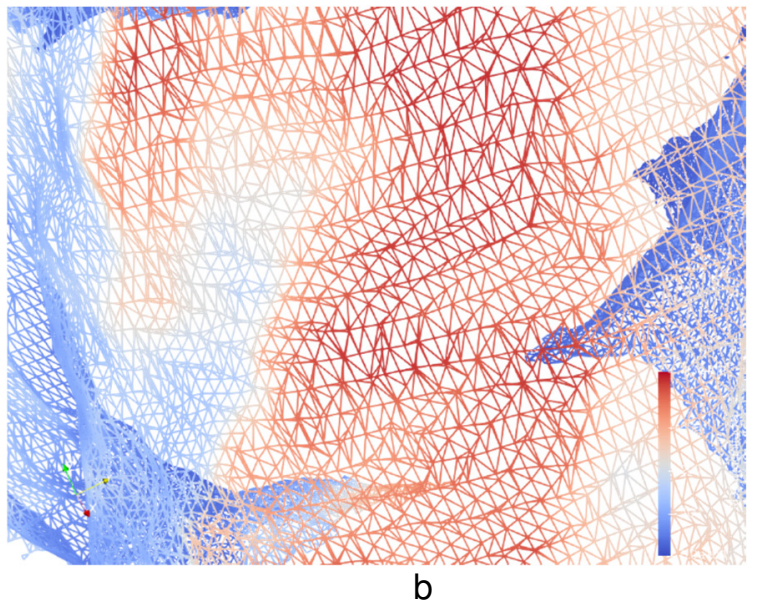

b

Fig. 1. Data after scan a) Unprocessed (raw data format) coordinates after 3D scan b) triangulated mesh over the points

Most of the modern scanner suppliers have good quality mesh processing software that provides a final triangulated mesh size for each scan. (Fig. 2, taken by IBV Scanner Move 4D). Such a good quality mesh is currently possible for people wearing body-hugging clothing and is generated on the basis of specially developed algorithms. In the case of scanning people with clothes and some additional parts (chairs, bicycle or other equipment), the mesh must be additionally processed to become suitable for generation of 3D volume meshes for Finite Element Analysis (FEM).

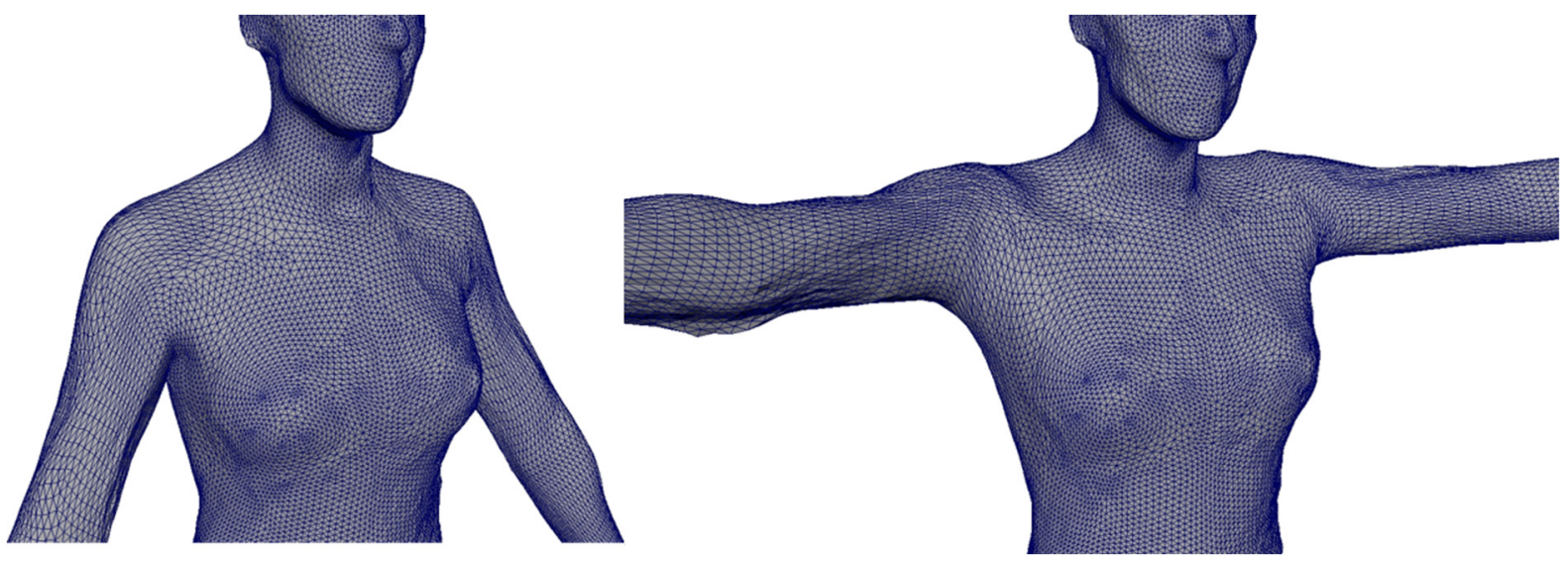

Fig. 2. Mesh in two poses after processing, obtained with IBV Scanner MOVE4D

\subsection{Mesh regularity and size}

The density of the scan mesh may vary in some areas of the scanned surface (Fig. 3a). The differences can be caused by the different geometry of seems, surface irregularities of coarser knitted structures, tapes of underwear or other accessories. The relief of the surface is often recognised by the algorithms and placed on these areas as more points in order to get the required accuracy of the geometry. Another place for getting differences in the distance between the points can be the areas where the boundaries of the single 3D raw images are located. In this case, a remeshing step is required, as explained in [6] for the case of adaptive refinement or in [7] for the usual cases. Figure $3 b$ shows the surface geometry of the body from Part $3 a$ after remeshing in order to get geometry with triangles with larger size, so that the computing time for the simulation remains acceptable. 


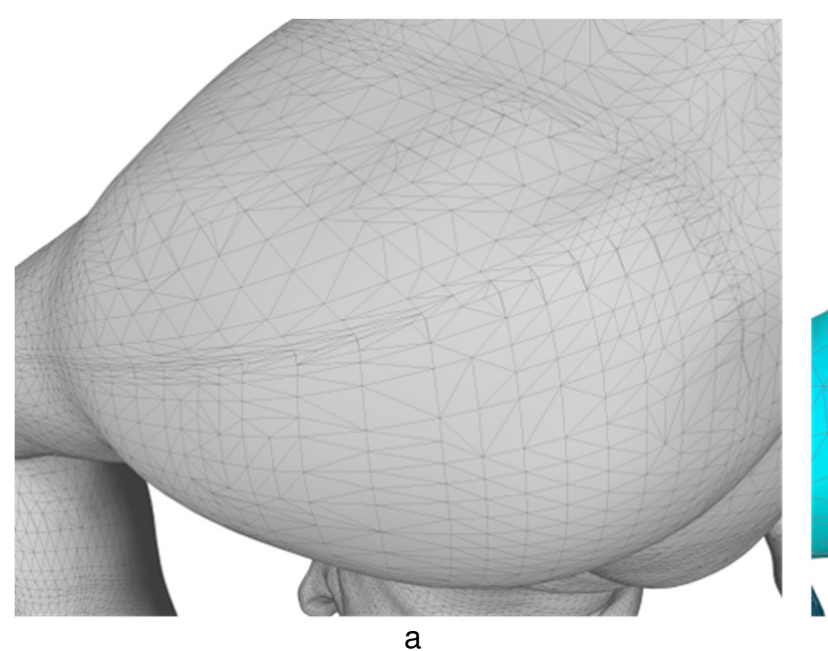

a

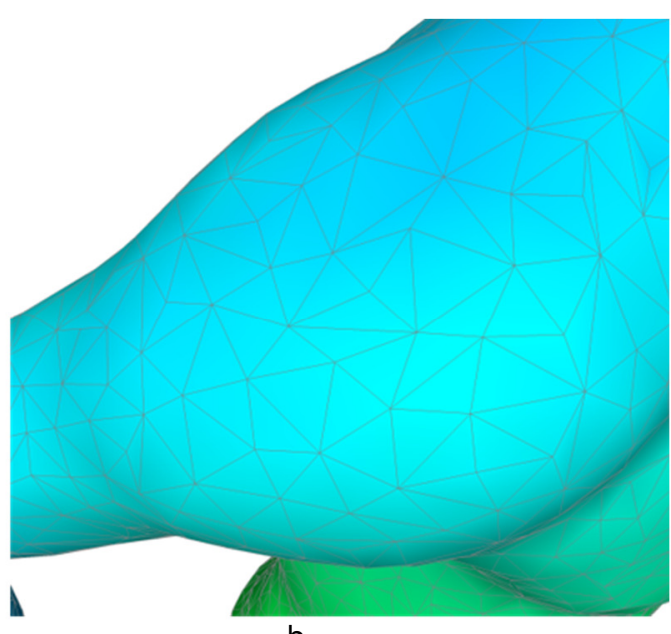

b

Fig. 3. The processed mesh from some scanners contains areas with different size a). After remeshing the geometry can be represented by elements with regular size for simulations

\subsection{Non-manifold meshes}

In several situations the generated mesh has defects in some places which disturb the next processing steps. There are different artefacts in the meshes, which represent non-existing in the reality configurations, named non-manifold meshes. These defects can be due to various reasons:

a) bugs or errors in the algorithms for creation of the triangle mesh based on the point cloud

b) bugs or errors in the remeshing procedures

c) problems of some manual processing such as selecting and cutting parts of the scanned object

d) additional objects or light, which reach the focus of the cameras during the scanning process.

Figures $4 a$ shows a case of non-manifold mesh, where three surfaces have a common edge. Such a situation cannot exists in reality for the description of a single body. The middle edge in this case hides the third one and such defects cannot be detected optically, but in some cases the edge crossing the existing mesh can be detected optically (Fig. 5a and Fig.5b).

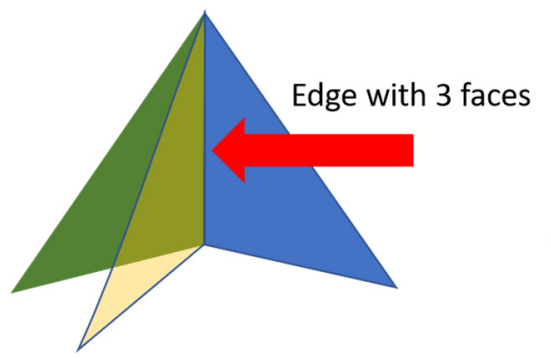

a

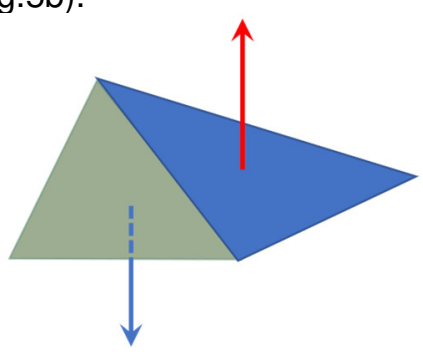

b

Fig. 4. Mesh defects. A) Edge with 3 faces and b) swaped normal vector.

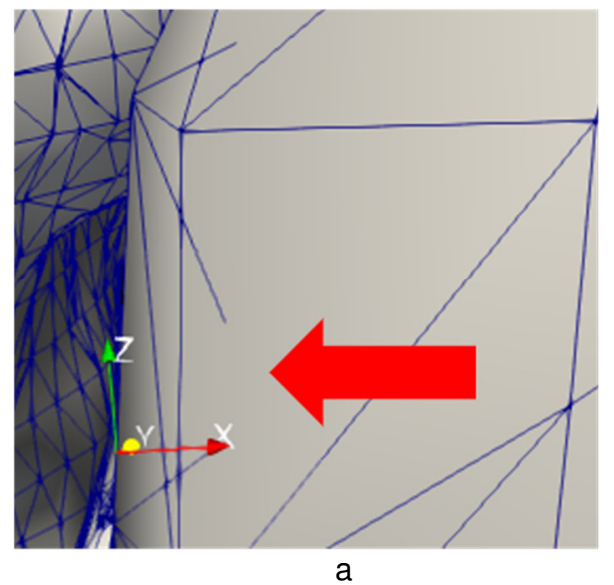

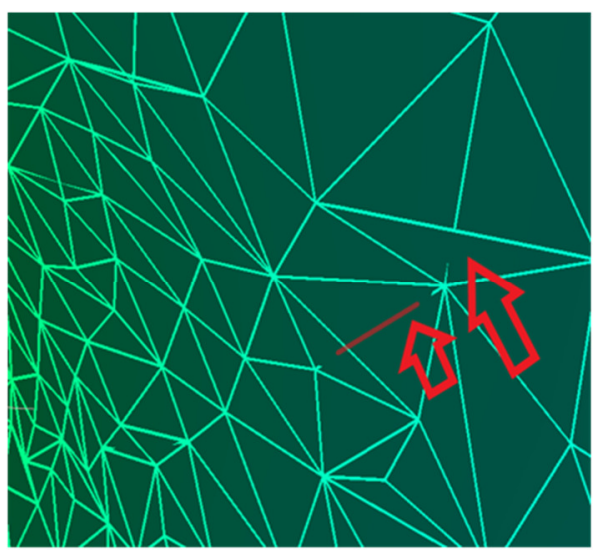

b

Fig. 5. Edges trough the surface, based shared common edge for three faces. 
The number of the surfaces connected to an edge can be counted in a software programme and the problematic edge can be listed and visualized (Figure 6b). Normally, such cases appear in geometries with sharp edges (Figure 6a). If the library or software used is not capable of automatically solving such a problem, manual inspection may be necessary to find and delete the artificial face.

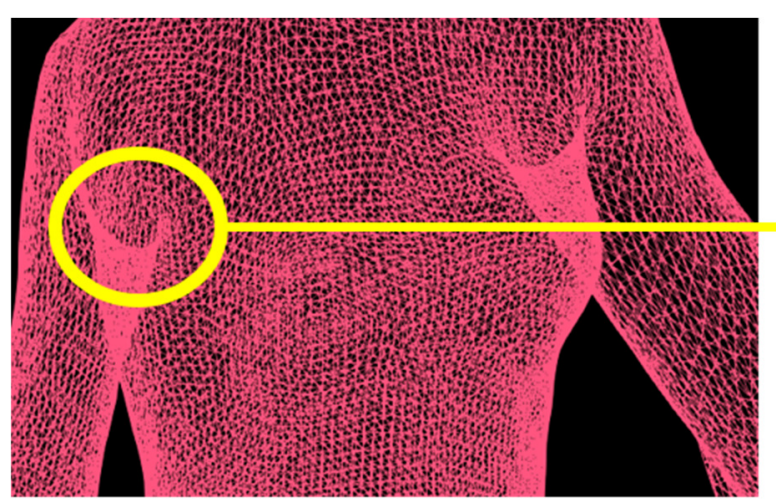

a

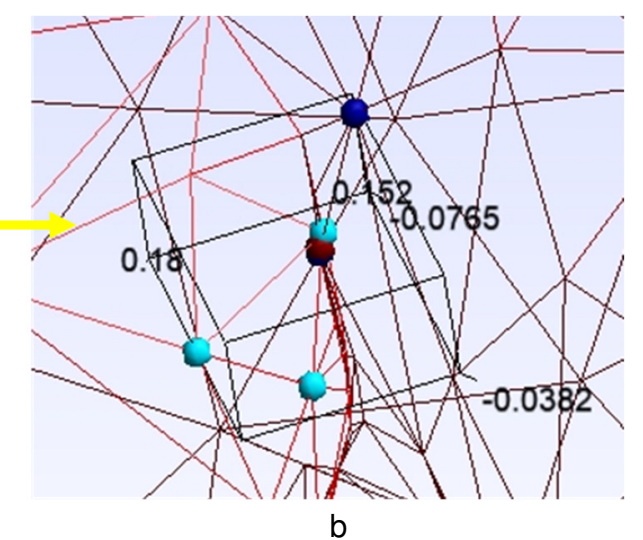

$\mathrm{b}$

Fig. 6. Edges trough the surface, based shared common edge for three faces.

\subsection{Missing connectivity}

Human hands and feet can be very close to the body in some poses and can form regions that cast shadows for the cameras trough the 3D scanning process. Poor lightning conditions or accessories on clothing can be another reason for missing surface information in certain areas. In this case, single elements without good connection to their neighbors can be created, such as surfaces with a common vertex, but without connecting another surface (Fig. 7) or a single surface in space. Some scanners, such as the Leo hand-held scanner of the company Artec [8],[9], recognise areas with not enough information and mark them in red during scanning so that the operator can get closer to them or scan from a different angle until enough information is connected. In contrary,scanners with multiple cameras take an image at all and do not have the option of requesting additional information for some areas, so open holes (Fig. 8b) or separate bodies (parts of accessories) may appear on the scan (Fig. 8 A). One simple rule for detecting such elements within a software algorithm is to count the areas to an edge. If an edge belongs to only one surface, it is not well connected and represents a hole. Such a test is integrated in the Meshlab software, for example [10]. It has a filter for detecting and closing holes, which requires information on the size of the hole to be closed.
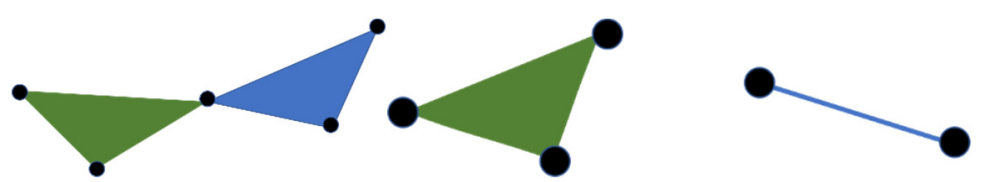

Fig. 7. Geometry with missing connectivity - not enough connected surfaces, single face or single edges and points

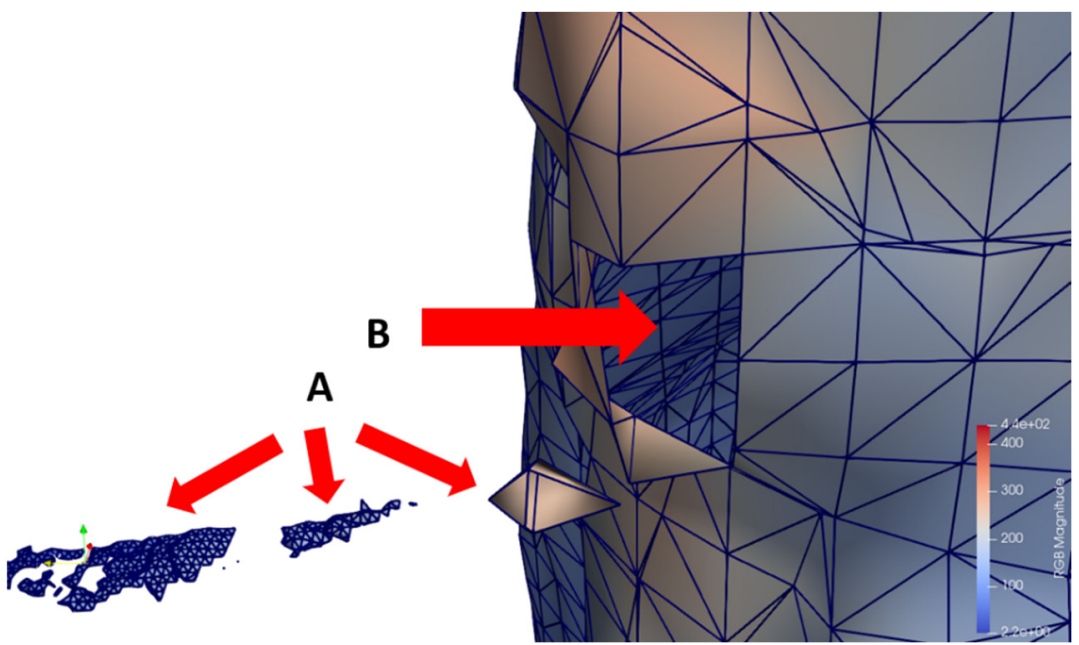

Fig. 8. Loosed connectivity of the mesh. A) separated regions b) open hole. 


\section{Example workflow from 3D scan to FEM}

The workflow for automatic creating FEM model includes the following steps:

a) load the scan data and create triangulate mesh (if not existing)

b) clean the mesh from non-manifold elements (remove surfaces on triple edges, close holes, delete separate geometry)

c) remesh with wished mesh size

d) fill in the surface with solid elements.

After this step, the material properties and the boundary conditions at the different elements has to be applied and the load case can be solved. Figure 9 shows some of these steps for a scanned geometry from a human leg, pressed with a fist, using in this case the linear implicit solver Z88 Aurora [11]. For realistic simulations of the human body, the elements has to be divided into groups corresponding to the different materials such as bones, muscles, skin and because of the low elasticity of the muscles, non-linear solver has to be used. Such investigation is planned as the next step. In the current example the goal was only to check the validity of the generated mesh based on a simple classical FEM simulation.

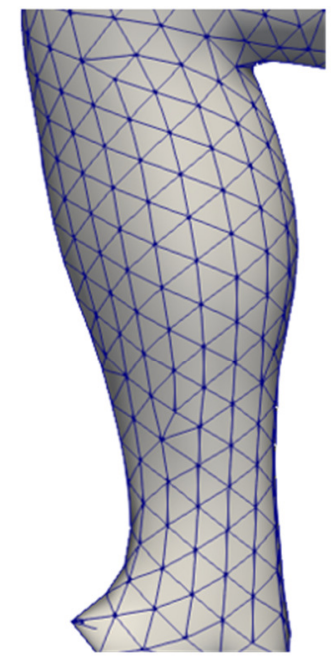

a

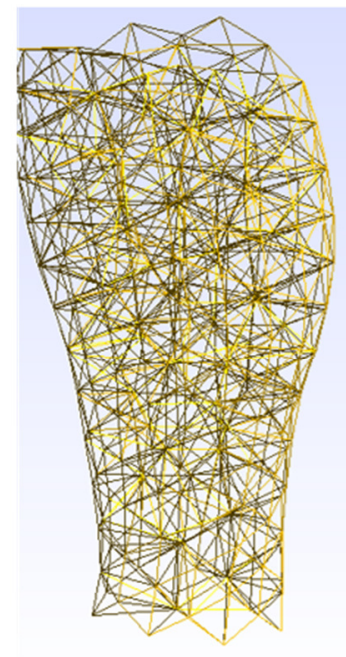

b

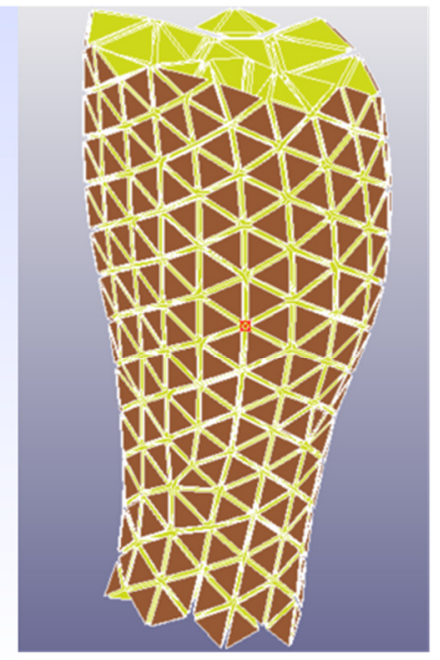

C

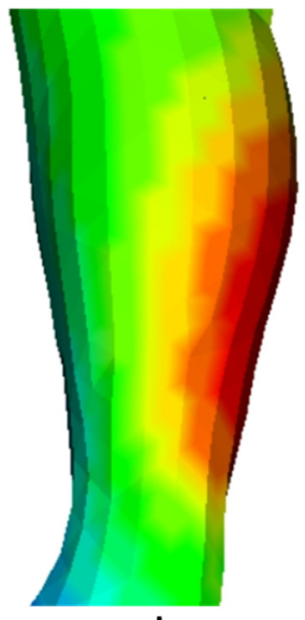

d

Fig. 9. From scan to FEM. A) surface mesh, b) and c) solid mesh d) deformation field after application of pressure on small area

\section{Special figures and motion}

The described in the previous section workflow works for a statical mesh of the human body. The 4D scanning technique allows several 3D images to be captured during human movement with high accuracy. Such data can also be used to verify simulations, but their processing is not so simple. For instance - the movement from sitting to chair to standing (Fig. 10a) can be analised during scanning, but the chair, the human body and the clothing represent a cloud mesh. The processing software is currently not able to separate these objects into separated groups and the analysis of such data has to be prepared for each case of movement individually. Another issue is the pretensioning of clothing (Fig. 10b). Not all parts of the clothing are in a relaxed state on the body, and some areas show a certain degree of pretension (Fig. 10b). If this frame is taken as the basis configuration, the information about the pretension has to be transferred to the load case of the finite element mesh. In the current state this procedure is not implemented. 

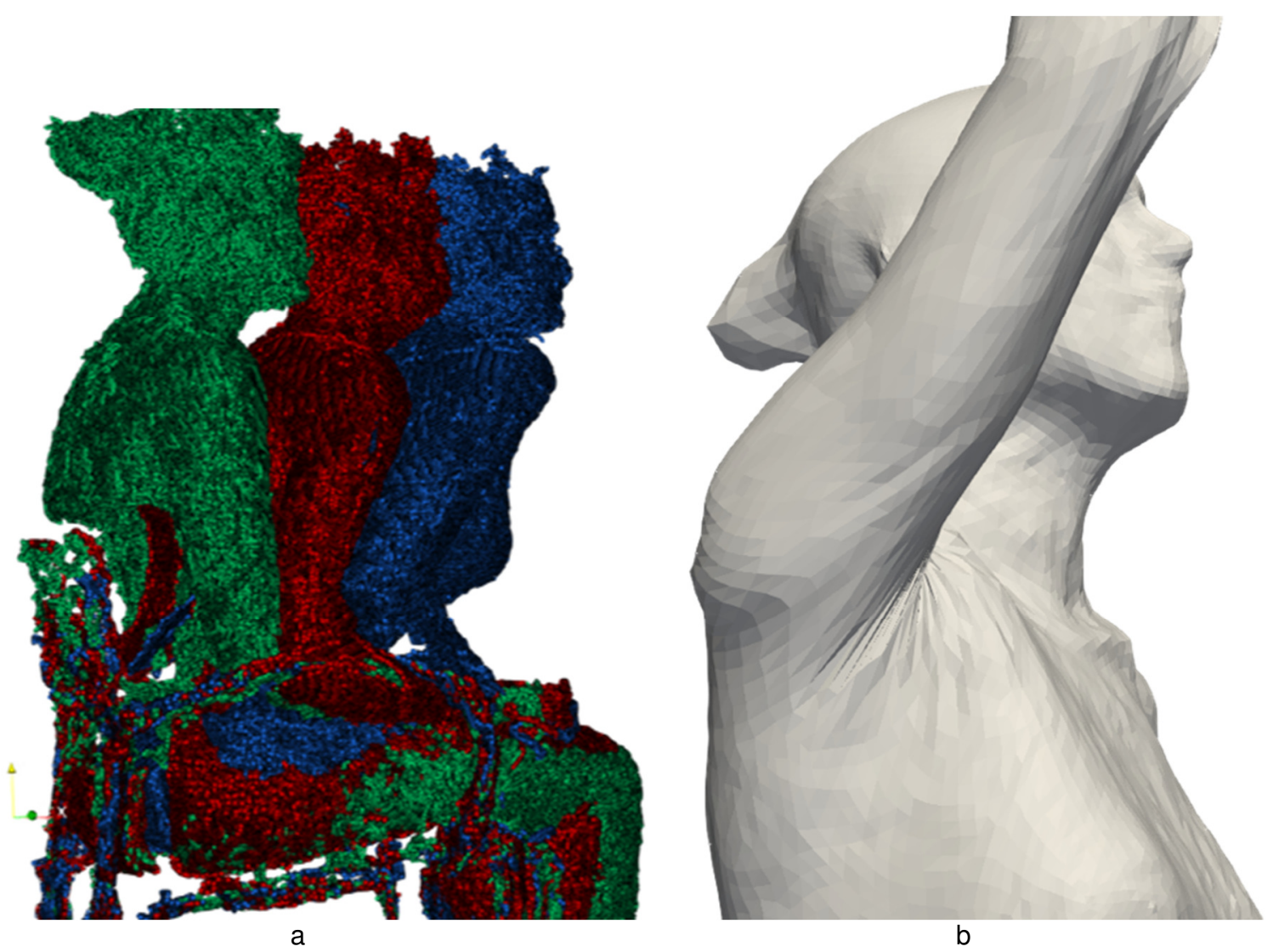

Fig. 10. Problem during the moving bodies: a) Raw data points from three motion steps include the chair geometry b) the stretching of the textile under the arm is visible, but the mesh does not contain the information about the initial state.

\section{Conclusions}

Presented are the common mesh defects, which have to be cleaned before a raw data set from the 3D scanner can be sent to the FEM meshing process to generate a surface and then a solid model of the human body. Some non-manifold geometries and missing connectivity resp. holes are among the common problems of the scanned meshes. After this has been solved and the surface has been remeshed with elements of normal size, it can be used in standard FEM software as a starting point for future simulations or extensions with different material properties.

\section{Acknowledgment}

The authors are very grateful to IBV - Instituto de Biomecánica https://www.ibv.org/ for their cooperation in preparing the 4D scans.

\section{References}

[1] C. Maurath, S. Gha, and et.al, 'Overview of LSTC's LS-DY NA An thro po mor phic Mod els', presented at the 11th In ter na tion al LS-DY NA Con fer ence, Michi gan, Daerborn, Jun. 2010.

[2] C. Zhihua and et al., 'A three-dimensional finite element modelling of human chest injury following front or side impact loading', Journal of Vibroengineering, vol. 18, no. 1, pp. 539-550, 2016.

[3] D. Harrison, Y. Fan, E. Larionov, and D. K. Pai, 'Fitting Close-to-Body Garments with 3D Soft Body Avatars', in Proceedings of 3DBODY.TECH 2018 - 9th International Conference and Exhibition on 3D Body Scanning and Processing Technologies, Lugano, Switzerland, 16-17 Oct. 2018, Lugano, Switzerland, Oct. 2018, pp. 184-189, doi: 10.15221/18.184.

[4] M. Loper, N. Mahmood, J. Romero, G. Pons-Moll, and M. J. Black, 'SMPL: A Skinned Multiperson Linear Model', ACM Trans. Graph., vol. 34, no. 6, Art. no. 6, Oct. 2015, doi: $10.1145 / 2816795.2818013$. 
[5] F. Bogo, A. Kanazawa, C. Lassner, P. Gehler, J. Romero, and M. J. Black, 'Keep it SMPL: Automatic Estimation of 3D Human Pose and Shape from a Single Image', arXiv:1607.08128 [cs], Jul. 2016, Accessed: May 19, 2020. [Online]. Available: http://arxiv.org/abs/1607.08128.

[6] A. R. Hermosillo, M. P. Romo, R. Magaña, and J. Carrera, 'Algoritmo de remallado automático de elementos triangulares durante los análisis con elemento finito', Rev. int. métodos numér. cálc. diseño ing., vol. 34, no. 2, May 2017, doi: 10.23967/j.rimni.2017.5.003.

[7] C. Geuzaine and J.-F. Remacle, 'Gmsh: A 3-D finite element mesh generator with built-in pre- and post-processing facilities: THE GMSH PAPER', Int. J. Numer. Meth. Engng., vol. 79, no. 11, pp. 1309-1331, Sep. 2009, doi: 10.1002/nme.2579.

[8] '3D-Objektscanner Artec Eva| Der Beste Strukturlichtscanner', Aug. 08, 2019. https://www.artec3d.com/de/portable-3d-scanners/artec-eva (accessed Aug. 08, 2019).

[9] Artec3D, 'Artec Leo'. [Online]. Available: https://www.artec3d.com/de/portable-3d-scanners/artecleo.

[10] P. Cignoni, M. Callieri, M. Corsini, M. Dellepiane, F. Ganovelli, and G. Ranzuglia, 'MeshLab: an Open-Source Mesh Processing Tool', in Eurographics Italian Chapter Conference, 2008, doi: 10.2312/LocalChapterEvents/ItalChap/ItalianChapConf2008/129-136.

[11] F. Rieg, R. Hackenschmidt, and B. Alber-Laukant, Finite Elemente Analyse für Ingenieure: Grundlagen und praktische Anwendungen mit Z88Aurora, 5., überarb. Aufl. München: Hanser, 2014. 